\title{
ABOUT EXTERNAL GEOGRAPHIC INFORMATION AND KNOWLEDGE IN SMART CITIES
}

\author{
R. Laurini ${ }^{\mathrm{a}}$, F. Favetta ${ }^{\mathrm{b}}$ \\ aKnowledge Systems Institute, USA, Roberto.Laurini@gmail.com \\ bLIRIS, Université Claude Bernard Lyon 1, France, Franck.Favetta@liris.cnrs.fr
}

\begin{abstract}
KEY WORDS: Geographic Information, Geographic Knowledge, Smart Cities, Territorial Intelligence, External Knowledge, Geographic Information Systems, Geographic Reasoning, Geographic Information Science, First Law of Geography.
\end{abstract}

\begin{abstract}
:
Any territory can easily be considered as an open system in which external effects can greatly influence its evolution in addition to inner dynamics. However, in practically all local authorities, their so-called geographic information or knowledge systems are bounded by the jurisdiction's limit, and therefore are closed systems. In this paper, we advocate the necessity not only to consider but also to include external influences within any GIS or GKS. Therefore, among external influences, we will consider beyond intra muros knowledge, extra muros knowledge divided in two categories, nearby neighboring knowledge, for instance located in an outer crown around the jurisdiction territory, but also farther knowledge for instance from technology watch. After having analyzed the semantics of borderlines, we suggest some element for the design of the crown and we analyze how the various components of a geographic knowledge base (objects, relations, ontologies, gazetteers, rules, etc.) can be integrated. Then some aspects regarding updating external knowledge are rapidly sketched. As a conclusion, we evoke the necessity of designing administrative protocols so that administration can negotiate the exchange of external knowledge bunches. In other words, this is an attempt to fully integrate the so-called Tobler's first law of geography.
\end{abstract}

\section{INTRODUCTION}

We are now entering into the knowledge society and local authorities must reorganize in order to base their behavior on knowledge. It can come from various sources, from experts to lay-citizens, not only by considering knowledge accumulated from centuries or millennia but also more recent knowledge bunches extracted from data mining.

Generally speaking, local authorities and especially cities have built information systems for planning and managing. But usually their data are describing various aspects inside their own jurisdiction (intra muros information). Existing computerbased maps illustrate perfectly this issue since outside information is not taken into account and generally neglected. But sometimes, external knowledge (extra muros) can be very important to understand the behaviour and the evolution of the zone corresponding to the jurisdiction taking external influences into account.

\subsection{General evolution}

Table 1 depicts the evolution. In the past, the distinction between storing and presenting geographic information was not very clear; but usually maps were drawn in a rectangle showing not only intra muros information but also extra muros information limited inside this rectangle allowing some kind of continuity for human reasoning. Then in the 60-70s, computer maps and GIS were incepted, but practically always storing data inside the jurisdiction; as a consequence, human reasoning and limited computer reasoning (such as spatial analysis) should be possible inside the jurisdiction but not outside. In the same period, satellite images and aerial photos appeared with two characteristics, (i) the borders were not visible and (ii) reasoning was not limited inside the jurisdiction. Now, by considering external information and knowledge, continuity of reasoning can be ensured especially within an out-buffer named also neighboring crown.

\begin{tabular}{|l|l|}
\hline $\begin{array}{l}\text { Conventional } \\
\text { manmade map }\end{array}$ & $\begin{array}{l}\text { The city is delimited, } \\
\text { included within a } \\
\text { rectangle a little bigger } \\
\text { than the usual } \\
\text { Minimum Bounding } \\
\text { Rectangle. } \\
\text { Continuity ensured } \\
\text { within this rectangle. } \\
\text { No automatic reasoning } \\
\text { allowed with vicinity }\end{array}$ \\
\hline $\begin{array}{l}\text { GIS-based } \\
\text { map }\end{array}$ & $\begin{array}{l}\text { The city is an "island" } \\
\text { located in a void space. } \\
\text { No continuity. } \\
\text { No automatic reasoning } \\
\text { allowed with vicinity. }\end{array}$ \\
\hline Satellite image &
\end{tabular}

Table 1. Various cases. 


\subsection{About geographic information and knowledge}

As data are essentially bits describing raw numbers, strings of characters, image or video pixels, information confers a meaning to data. Knowledge attaches purpose and competence to information and can generate potential actions. Another way of defining knowledge is to claim that knowledge is information useful to solve a problem. More generally by using knowledge adequately, this is the beginning of wisdom. A very important aspect of knowledge is formed by IF-THEN rules.

As knowledge becomes currently used in business often under the name of business intelligence, it seems important to create an infrastructure so that local authorities tend toward the socalled territorial intelligence. As Dangermond (2010) said "As we move from an industrial economy to a knowledge-based economy, our reliance on physical infrastructure is being supplemented by reliance on knowledge infrastructure, of which geographic knowledge will form a key component".

More generally "geographic knowledge corresponds to information potentially useful to explain, manage, monitor and plan a territory" (Laurini, 2017).

Figure 1 gives the structure of a geographic knowledge base system (GKS) in which components will be detailed farther in this paper. One of them is external knowledge.

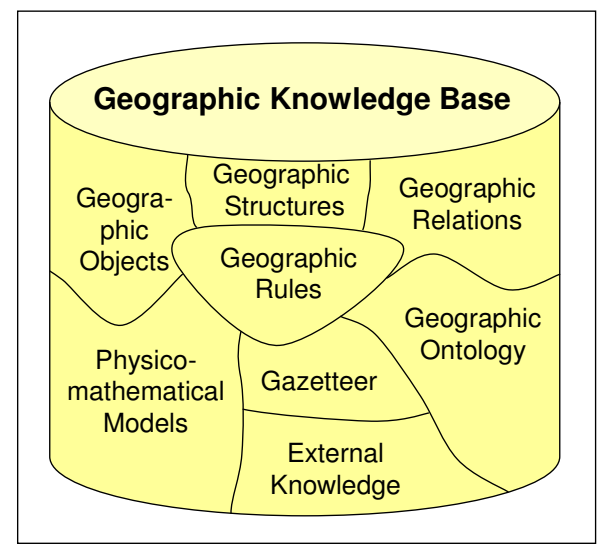

Figure 1. Components of a Geographic Knowledge System.

\subsection{External geographic knowledge}

As previously told, usually GIS are limited to data inside their jurisdiction. But external influences must be taken into account. Let us examine a few examples.

- Suppose any human settlements, even small through which a big highway is crossing; the influence of road traffic is very important to understand and manage this settlement.

- Suppose a city with a big river; any climatic conditions upstream must be integrated to understand possible floods.

- Suppose a huge shopping mall located in the neighbouring crown; it is an important actor not only for consumption, but also as a set of employees; journeys for working of for services will affect also local traffic.

- Within a town, suppose an industrial plant owned by a multinational trust; its decisions made perhaps from another continents can be drastic for the town's evolution.

- Suppose a city with an important railway station or even an airport: some nation-wide problems can have outcomes on traffic.
- Consider for example the diffusion of bike rental systems; by technology watching; various cities have decided to implement this kind of system for various reasons, $(i)$ to easy urban traffic, (ii) to decrease pollution and (iii) to increase the level of physical exercises for humans.

Remember Tobler's law (Tobler 70), often called as the first law in geography: "Everything is related to everything else, but near things are more related than distant things". It implies not to limit to the jurisdiction, but also to consider external influences, in other words, external knowledge.

So let's define external knowledge as bunches of knowledge concerning external influences important for a territory. Two categories will be examined:

- Short distance or neighbouring knowledge, i.e. knowledge located at the vicinity of the jurisdiction; a solution can be to determine of out-buffer zone around the jurisdiction and consider knowledge inside this buffer zone. In Cherni (2015), a rule of thumb proposes that the width of the out-buffer must be stated as $D=\sqrt{S} / 20$, in which $\mathrm{S}$ denotes the area of the territory.

- Long distance knowledge includes the source and contents of external influences including urban technology watching.

\subsection{Organization of the paper}

The objective of this paper is not to present a prototype of a geographic knowledge system integrating external knowledge, but only to clarify this concepts and analyse a few issues. So, this paper will be organized as follows. First the case of neighbouring knowledge will be studied starting from the semantics of jurisdiction's borderline in order to define different bunches of knowledge; and we will examine how to ensure the continuity of reasoning between outside and inside a jurisdiction. Then urban technology watching will be rapidly considered. To conclude this paper, some hints concerning the structuring and updating of external knowledge will be given.

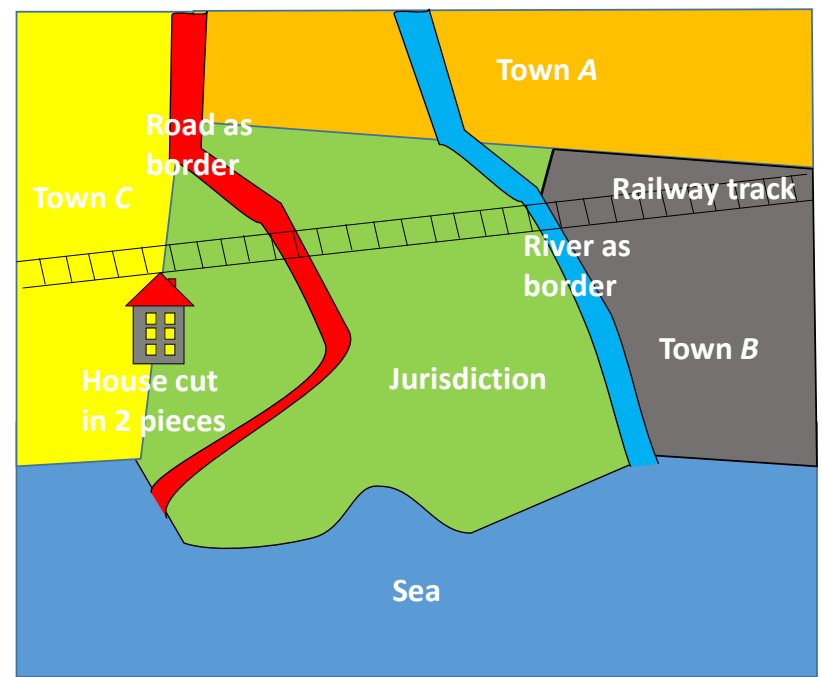

Figure 2. Several types of borderlines and geographic objects.

\section{SEMANTICS OF BORDERLINES}

For analyzing neighboring knowledge, it is important to examine borderlines. Indeed, two categories can be rapidly distinguished, natural and artificial borders. For natural borders, seas are perhaps the main elements. By artificial borders, we 
means land borders usually agreed-upon, but several cases must be studied. See Figure 2 for an example of problems.

\subsection{Land-sea borders}

A lot of countries have maritime borders. Here the concept of national waters must be considered for countries usually within 200 nautical miles. This aspect is important for fishermen especially when international treaties modify fishing rights, for instance as a Brexit consequence in UK and North-Western Europe.

An important issue deals with harbors which are the interface for maritime transportation. Any international crisis concerning this activity can impact the evolution of the port city.

Same considerations can be taken for huge waterbodies such as the Lake of Geneva, and the Nord-American Great Lakes.

\subsection{Borders in mountains}

Borders in mountains are also common. Usually, they really correspond to a physical barrier (see Andean Mountains between Argentina and Chile). But often passes allow traffic.

\subsection{Borders along rivers or roads}

Taking river axes as borderlines is also common (See St Laurent River between Canada and USA); as a consequence one bank is owned by a jurisdiction, and the other by a second one. Here considering neighbouring knowledge implies to store information relative to both sides. Sometimes, along roads, the border can be the axis of the road, but rarely one of its shoulder. For railway tracks, the problem is similar. In addition, if one jurisdiction wants to repair a road, it does not repair only the portion of the road belonging to it; here a good practice is to discuss with the neighbouring jurisdiction to organize the complete repair.

Here considering external knowledge implies to extend the storing of networks outside the jurisdiction's territory.

\subsection{Roads or rivers traversing}

Numerous rivers or roads go through jurisdictions. Let us call contact points the places where they come in and go out. Usually in conventional GIS, those contact points correspond to nodes in the road or in the river networks. Here also network extension must be taken into account.

\subsection{Other types of borders}

In a lot of places, borders are in the midst of countryside. Apparently no extra form of knowledge must be considered

\subsection{Conclusion regarding borderlines}

Whatever the cases are, all imply to consider not only geographic objects located in the neighborhood, but also the spatial relations they share, and perhaps other form of knowledge such as geographic rules

\section{ENSURING REASONING CONTINUITY THROUGH GEOGRAPHIC RULES}

Morgan (2008) and Ross (2011) have declared that rules shall be considered as first-class citizens in information technology and especially in business intelligence. And two sorts of rules were proposed: IF-THEN-Fact and IF-THEN-Action. By means of the first one, some new facts or attribute's values must be implied whereas though the last one some actions must be performed.

In Laurini (2016), some geographic rules were analysed in which the management of space must be modelled. In addition to so-called co-location rules (Shekhar-Huang 2001), this leaves to the design of rules such as IF-THEN-Zone. Here, a new kind of geographic rules must be considered with two different zones, one in the antecedent and one in the consequent, the prototype of which can be modelled as "IF something here, THEN something else there"; let us call them bi-location rules. It can also be considered as a cause-effect chain in which cause and effect are respectively in different zones.

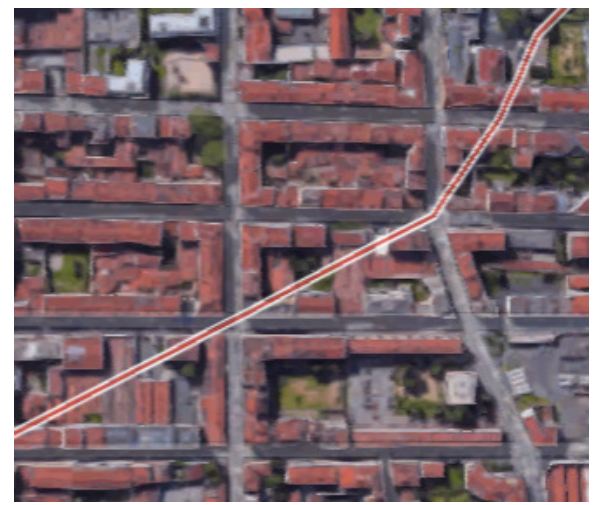

Figure 3. Example of borderline between two adjacent cities (Lyon and Villeurbanne) cutting city-blocks and buildings into two different parts.

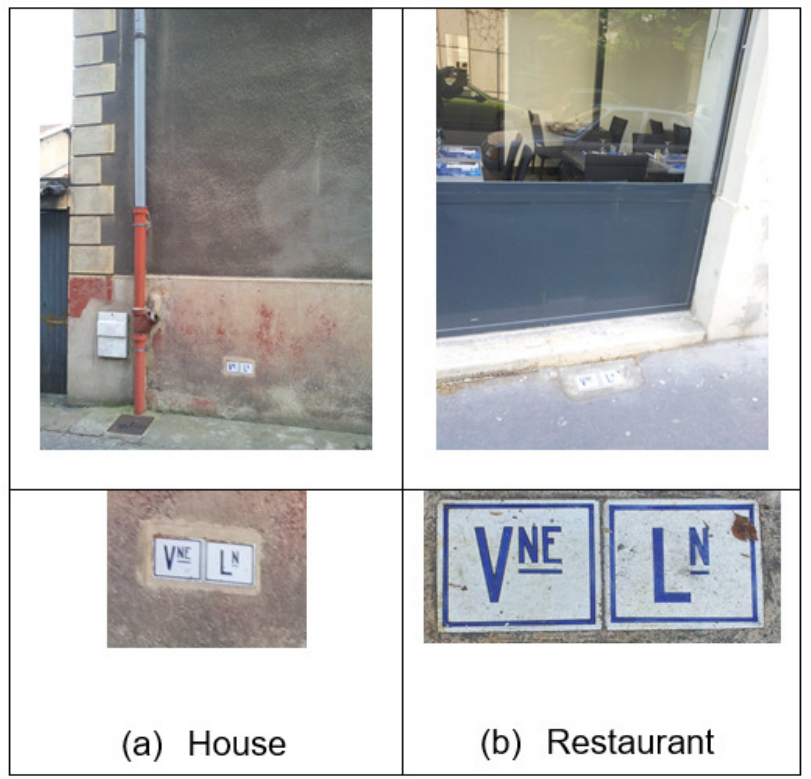

Figure 4. Example of urban objects split by administrative boundaries in which $\mathrm{V} \underline{\mathrm{NE}}$ and $\mathrm{L} \underline{\mathrm{N}}$ respectively means Villeurbanne and Lyon. (a) an house. (b) a restaurant.

Figure 3 shows a portion of the borders between the French cities of Lyon and Villeurbanne in France. And Figure 4 illustrates two urban objects split by administrative boundaries. Figure $4 \mathrm{a}$ gives the case of house and Figure $4 \mathrm{~b}$ for a restaurant in which a customer can ask a table either in Lyon or in Villeurbanne. 
Eventually, once the artificially cut features are reconstituted, three types of continuity can be defined.

- Object continuity: Sometimes geographic objects are artificially cut by borders. We know people having their house in which the kitchen is in one town and their bedrooms in another towns (see an example Figure 3). In this case, the complete objects must be stored perhaps with an indicator reading that a portion of the concerned object is subject of another jurisdiction. Similarly, big properties, mountains, marshes and recreational parks can belong to several jurisdictions.

- Network continuity: The case of networks is particularly interesting. Some aspects were already studied in Laurini (1998) in order to ensure seamless continuity between several federated GIS. Here the problem is similar, that is continuity of the edges and nodes (Figure 5)

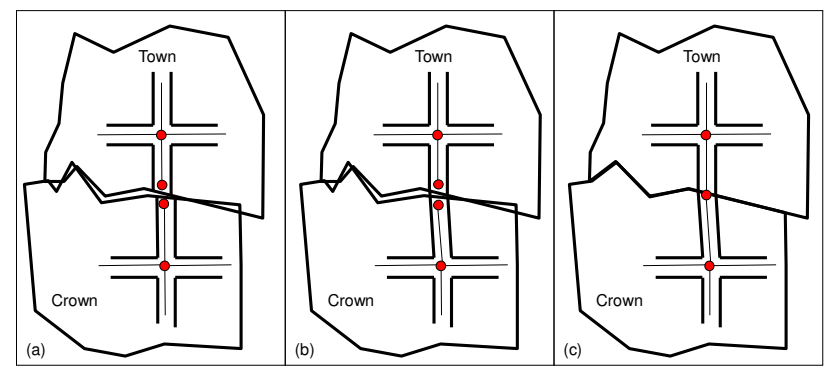

Figure 5. Network continuity between a town and its crown. (a) Preliminary situation with error discrepancies. (b) Merging roads so that the map looks good. (c) Merging nodes at the border.

Eventually, three levels of continuity can be identified:

1 - Geometric continuity: in which maps look good by correcting border discrepancies so that maps are extended to the neighbouring crown; but objects are not reconstructed in the knowledge base.

2 - Semantic continuity: reconstruction of geographic objects partly cut by borders such as buildings, parcels, etc.

3 - Topological continuity for networks: this will allow consistent graph reasoning (Figure 5c).

Now that semantics of borderlines are defined, let us examine remote information and knowledge which can influence the dynamics of a territory, namely technology and sociological watches.

\section{TECHNOLOGY AND SOCIOLOGICAL WATCHING FOR LOCAL AUTHORITIES}

For increasing the development of a smart territory, one interesting aspect is to try to benefit not only from new technologies, but also from others' experiences. In other words, technology watching must be completed by a kind of sociological watching integrating successful experiments made in other cities in order to take innovations into account coming from both companies and from other territories. Let us rapidly study each of them.

\subsection{Technology Watch}

The task of the technology watch is to observe, track, filter out and assess potential technologies from a very wide field extending beyond the normal confines of the sector. Often the expression "Technology Watching" is linked to "Competitive Intelligence" (Rovira 2008).

For instance, after Araujo Campos (2016), the "humble" lamppost can be transformed into an intelligent sensor for light, air pollution, noise, etc.

\subsection{Urban Sociological Watching}

A complementary aspect is to be aware of novel innovations in other cities and territories also from a sociological aspect. Facing a new transport-on-demand system established in the city $A$, it could be interesting to analyse the outcomes in this city; and based on this analysis, to examine whether this solution can be imported to another city $B$. But overall, a local decision-maker must imagine how this kind of system can be integrated.

\subsection{Diffusion of innovation in cities}

The generalization of the biking rental system in several cities can be seen as a result of both technologic and sociological watching.

Concerning the diffusion of innovation. According to (Rogers, 1995), the steps are as follows:

1) Knowledge - person becomes aware of an innovation and has some idea of how it functions,

2) Persuasion - person forms a favourable or unfavourable attitude toward the innovation,

3) Decision - person engages in activities that lead to a choice to adopt or reject the innovation,

4) Implementation - person puts an innovation into use,

5) Confirmation - person evaluates the results of an innovationdecision already made.

In our case, the first problem is to describe an innovation as knowledge. Let us call it innovative knowledge. Then a subsequent problem, outside the objective of this paper, will be to introduce it into a reasoning mechanism. Don't forget that perhaps a very novel innovation made within the territory under jurisdiction can appear as innovative knowledge elsewhere.

\section{ORGANIZING EXTERNAL NEIGHBOURING KNOWLEDGE}

The first aspect is to put a marker indicating knowledge chunks as either internal, neighbouring or innovative knowledge. Then we must delimitate the neighbouring crown. And finally, let us examine various geographic knowledge components but setting aside the case of innovative knowledge. See (Laurini 2017) for more details concerning chunks of internal knowledge because often, the way of modelling could be not very different. In this section, only the main differences will be studied.

\subsection{Crown delimitation}

How big the crown must be? Two possibilities appear, either with a regular width or with an irregular one in connection with requirements. 


\subsubsection{Regular width}

To start the discussion, let us consider a circle (Figure 6) whose area $S=\pi r^{2}$ and another circle with the same centre whose area is $S^{\prime}=\pi(r+d)^{2}$ in which $d$ is the width of the out-buffer.

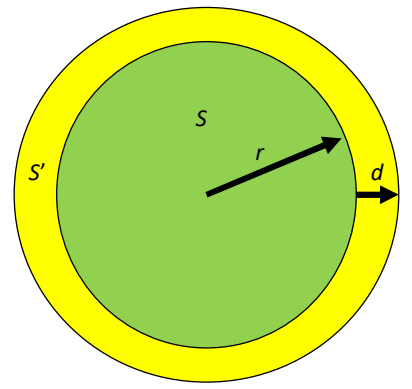

Figure 6. A circular territory with its out-buffer crown

Let us pose $S^{\prime}=(1+\alpha) \times S$ in which $\alpha$ represents the coefficient, for instance $10 \%$ of the area of the country. We can easily deduce:

$$
r+d=r \sqrt{(1+\alpha)}
$$

So giving

$$
d=\sqrt{\frac{S}{\pi}}(\sqrt{1+\alpha}-1)
$$

For instance, take a city $100 \mathrm{~km}^{2}$ wide and a crown corresponding to $\alpha=20 \%$, the out-buffer size will be $538 \mathrm{~m}$ wide.

Even if all territories are not roundish, this formula can be taken as a preliminary rule of thumb to define the width of the crown.

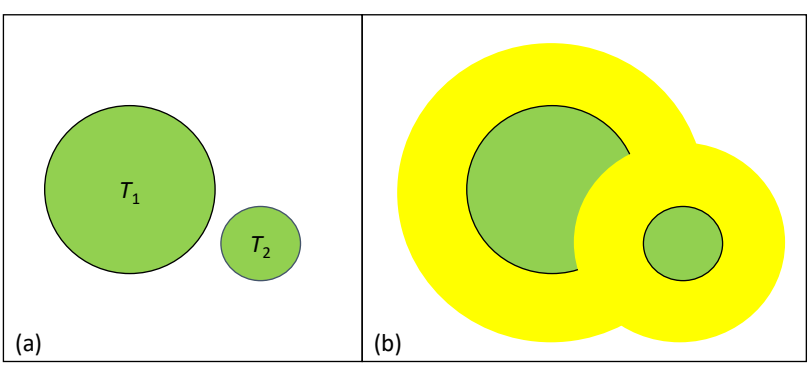

Figure 7. Case of non-connected territories. (a) A territory with two components. (b) With their outer crowns, each with the same width.

But when we have to deal with a non-connected territory, i.e. for instance with many islands, the problem is a little bit more complex. Two solutions seem possible:

1 - Delimit the crown around each part of the territory with the same width;

2 - Compute an out-buffer for each components; in this case as depicted Figure 7, $T_{1}$ 's crown overlaps $T_{2}$ 's one.

Consider for instance USA whose area is about $9,800,000 \mathrm{~km}^{2}$. For $\alpha=20 \%$, the distance is $167 \mathrm{~km}$. As for the conterminous states, this value is acceptable, what about the various US territories scattered in the Pacific or elsewhere such as Guam or Guantanamo? A different value can be assigned to smaller territories.

\subsubsection{Irregular width}

Another possibility is to decide to decide the crown width irregular. In this context, what could be the rules to define it?
For instance, let us start with a regular width as a first crown delimitation. Of course, if there is nothing important, one can decrease the width, and when there are interesting features (shopping mall, important plant, important recreation zone, etc.), the crown width can be extended. Moreover, the width can be modified if necessary (Figure 8).

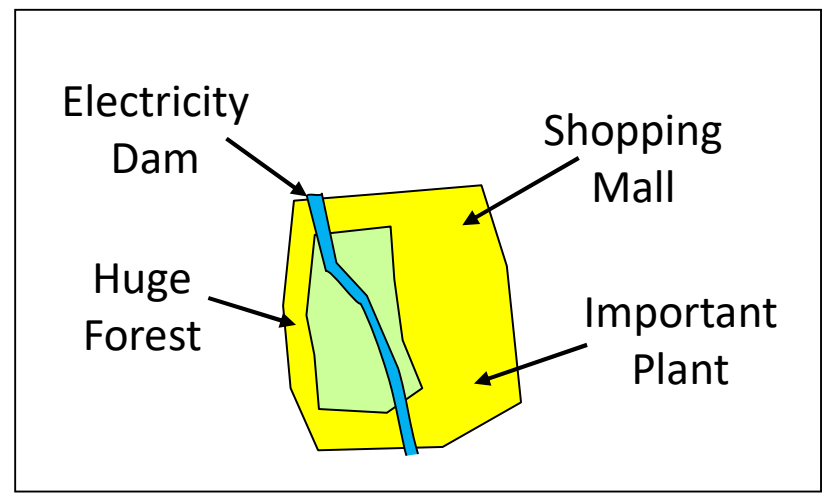

Figure 8. A circular territory with its out-buffer crown

An important aspect to consider is linked to cost. It is obvious that the wider the crown, the more expensive is the management and updating of external information and knowledge. This issue, based on a cost-benefit analysis, must be taken into account when deciding the width of the crown.

\subsubsection{Contents based on requirements}

Another way is to determine first the contents of the crown and then its width. Indeed, a good practice should be to start from the requirements as defined for the design of the information or knowledge system in a local authority, by asking the following question: "what are the geographic features and phenomena outside the jurisdiction which can affect the evolution and the management of the jurisdiction's territory?"

For answering, a solution can be to make a survey at the vicinity of the jurisdiction and to consider the objects which can affect the dynamics of the territory, so defining the crown.

\subsection{About influencing geographic objects and phenomena}

Within the crown, some geographic objects can have little influence, but farther some other do. In Figure 9, some cases are shown. Geographic objects such as $R$ as divided in two parts; similar to $S$, the objects have a portion inside the crown and outside. They will be considered only if its influence is agreed. Farther objects such $W$ will be included contingent upon its influences are considered as important. For rivers such as $U$, upstream, is more important, for instance for floods and pollution. Concerning roads such as $\mathrm{V}$, their outside portion must be considered in the case of important highways.

In conclusion regarding external influences, even if some rules can be stated to select objects or portions of objects to be included, pragmatism must help decide. 


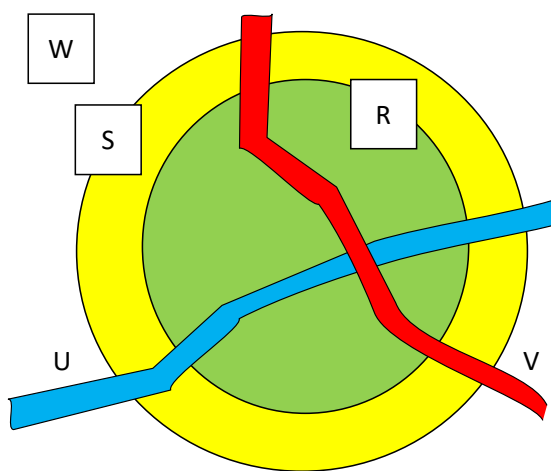

Figure 9. Location of some geographic objects able to influence jurisdiction's inner dynamics.

\subsection{Integration or separation}

Now that the outer crown is delimited, let us examine how to organize the geographic knowledge base. Two ways are possible:

- Total integration, i.e. all objects contained in the crown are included into the knowledge; based on this organization, when one wants only the objects within the jurisdiction, a clipping procedure with the border must be launched. As a consequence a single repository is need. However, there is a problem of updating; indeed as the updating within a jurisdiction is easy, for the objects within the crown, it is a little bit more complex since automatic procedures with neighbouring jurisdictions must be negotiated and run.

- Separation, i.e. partly cut objects are stored in two different repositories. In this case, reconstruction procedures must be invoked when necessary. However, it can be agreed that the degree of quality (updating) can be different.

In this text, only the second way (separation) will be studied. So, the question is "how to link the components of the two repositories?"

\subsection{Links between repositories}

In this section, only the consequence of separating the two repositories will be examined.

\subsubsection{Neighboring geographic objects}

As previously told, two categories must be taken into account in the crown, those totally included and those astride the borders. As a consequence, two possibilities. The first one will be, at the creation of the knowledge base, to complete the objects with their components in the crown or elsewhere. The second will be to consider separated the different components together with technical rules to re-construct them:

- Rules to reconstruct partly-cut areal objects (houses, recreational parks, etc.);

- Rules to reconstruct ribbon objects (as rivers and roads) and then to reconstruct their networks. For details refer to (Laurini 2014, 2017).

To illustrate the principle, suppose a house cut into two pieces, the first one belonging to a city and the second to its crown. To simplify the writing of the rule, suppose in addition that there are no measurement error (for notation, see Laurini (20017) in which Egenhofer relations are used and $\Omega$-type correspond to an ontological class:

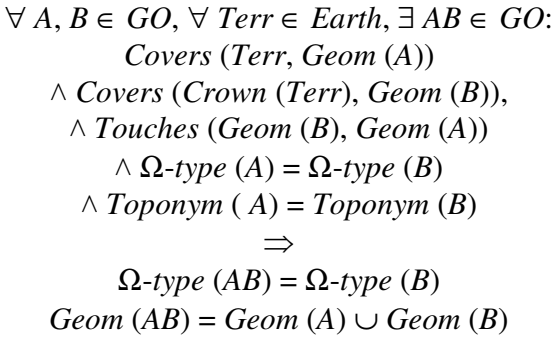

\subsubsection{Geographic relations}

New kinds of geographic relation must be integrated namely for objects astride the border and for objects traversing the crown. Consider a geographic object $O$ cut into two pieces $O^{1}$ and $O^{2}$, the model will be

$$
O=\left\{O^{1}, O^{2}, \text { border_astride }\left(O^{1}, O^{2}\right)\right\}
$$

\subsubsection{Geographic ontology}

The ontology must not be modified, except by adding the astride relation. However, several cases must be taken into account.

1 - If, in the crown, there is a territory with another language, classes in the outside ontology can differ.

2 - Even if the language is the same, the outside ontology can differ.

Anyhow, by taking those aspects into account, using a single ontology will easy geographic reasoning.

\subsubsection{Gazetteer}

The toponyms of places inside the crown or astride the border must be integrated into the gazetteer. The problem of a possible different language must also be taken into consideration.

\subsubsection{Applicative Geographic rules}

In addition to the previous rules, some general applicative rules must be considered as bi-location rules. Among them, as one of the location will be inside the jurisdiction, the second location could be either in the crown or outside else.

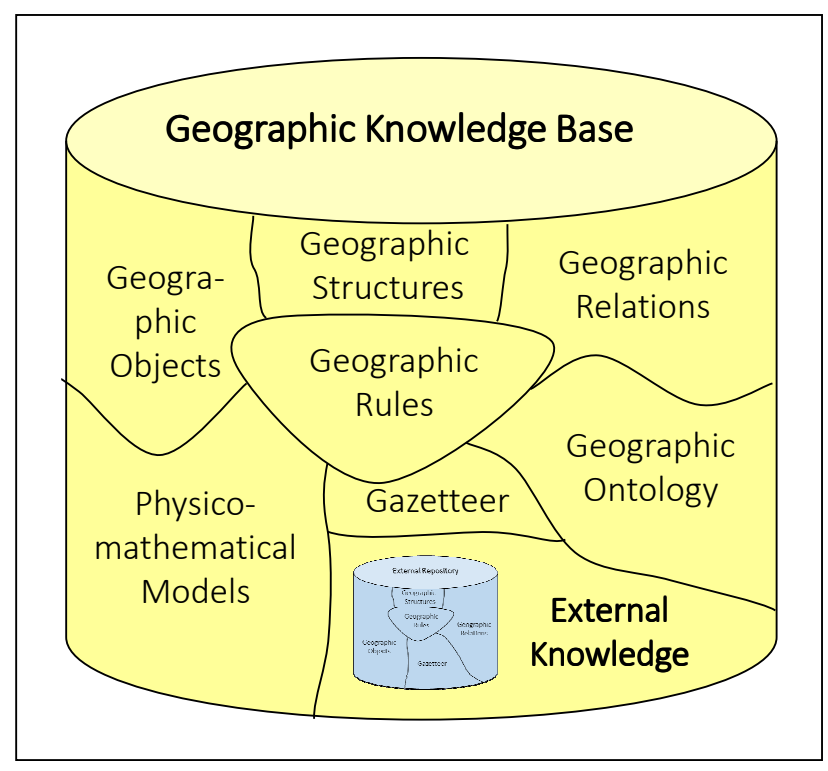

Figure 10. Architecture of a Geographic Knowledge Base with an additional repository for storing external knowledge. 


\subsubsection{Proposition of an architecture}

As previously told, two solutions seem possible. The first one is to keep the previous structure (Figure 1) by stating that the territory covered by the knowledge base not only includes the jurisdiction but also the external crown. In this case, the external repository only includes technology and sociological watching. Maybe, some dedicated metadata can help distinguish the crown's chunks of information or knowledge.

The second solution is to store external information in a separate repository as depicted in Figure 10. In this case, this separate repository will have a structure similar to the jurisdiction's knowledge base, but without components such as ontology and models which can be shared.

\section{UPDATING}

Now that the main characteristics are examined, a very important issue concerns the updating of the external information and knowledge and especially within the crown. Two facets must be studied, namely juridical and technological.

Consider a city having such a GKS with extra muros information and knowledge. Two solutions can be considered: (i) an urban planner must be in charge of examining the evolution of geographic objects within the crown, and as soon as there is a change, the concerned objects must be updated; (ii) a second solution is based on a negotiated protocol with the neighboring institutions in order to make updates automatically. Let us examine only the second solution.

By extending this idea, a neighboring town can also have a crown in the concerned jurisdiction as exemplified in Figure 11.

The solution is to design a trigger so that when an update is made within a crown of a territory $A$, an update is automatically made in the partner GKS $B$.

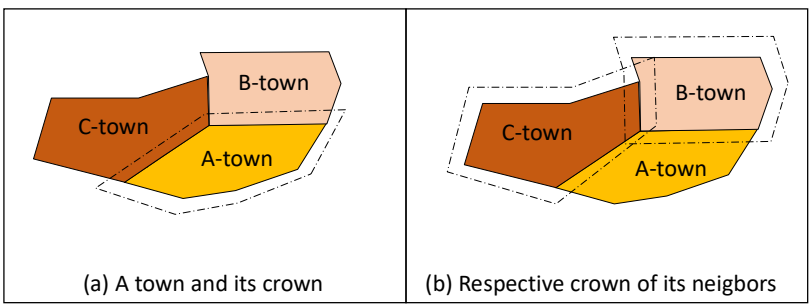

Figure 11. A town, its own crown and the crowns of its neighbors.

\begin{tabular}{|c|}
\hline When update $O$ in $\mathrm{GKS}_{\mathrm{A}}$ \\
$\{$ If $O$ belongs to Crown (TerrB) \\
Then update its counterpart in $\left.\mathrm{GKS}_{\mathrm{B}}\right\}$ \\
\hline
\end{tabular}

Similar triggers can be written for creating or deleting any geographic object belonging to a partner knowledge base.

An additional problem comes from privacy. Indeed, for some particular reason, a jurisdiction can decide not to offer neighbors some information or knowledge. This problem can be solved by defining some views (in the database sense) and/or by detailing it within the collaboration protocol.

\section{CONCLUSIONS}

In smart cities and territorial intelligence, it is of paramount importance to make geographic reasoning for planning or managing any territory and not to be blocked by borders.

So, the objective of this paper was to study the concept of external geographic information and knowledge, and to examine a few issues concerning their use to ensure continuity of knowledge reasoning, as involved in the first law of geography (Tobler's law).

For that purpose, it was necessary to study the dynamics of geographic objects located in the outskirts of a jurisdiction which can have influences into this jurisdiction such as roads, rivers entering or traversing a jurisdiction, or even important plants or shopping malls.

Therefore, an external crown of any territory must be delimited, and some rules have been given to determine its width, maybe regular or irregular based on a cost-benefit analysis.

Concerning remote influence, the importance of technology and sociological watch for urban activities have been rapidly sketched, but a comprehensive mechanism to model such issues are still missing.

Then, some aspects regarding updating of external geographic knowledge have been studied especially based on a protocol with neighboring jurisdictions and operated through triggers.

Among perspectives, let us mention, among others:

- To refine the analysis of external information and knowledge semantics and the ways to model them in geographic knowledge bases and to integrate them in any geographic reasoning;

- The writing of technical rules regarding external knowledge;

- The detailed modeling of geographic knowledge coming from technological and sociological watching;

- The protocols to define for the ongoing updating of the crown data, information and knowledge;

- And the definition of metadata relative to external information and knowledge.

\section{REFERENCES}

Araujo Campos J., 2016, Connected Cities, Integrated Cities. Key-note address presented at AFI $360^{\circ}$ Conference Track on Smart Cities and the Future Internet, May 25-27, 2016, Puebla, Mexico.

Cherni I. 2015, Découverte de chorèmes par fouille de données spatiales. PhD, September 11, 2015, INSA of Lyon, France and Institut Supérieur de Gestion de Tunis, Tunisia.

Dangermond J., 2010. Geographic Knowledge: Our New Infrastructure. Can be downloaded from http://www.esri.com/news/arcnews/winter1011articles/geograph ic-knowledge.html.

Laurini R. 1998. Spatial Multidatabase Topological Continuity and Indexing: a Step towards Seamless GIS Data 
Interoperability. International Journal of Geographical Information Sciences, vol. 12, 4, June 1998, pp. 373-402.

Laurini, R. 2014. A Conceptual Framework for Geographic Knowledge Engineering, Journal of Visual Languages and Computing (2014), Volume 25, pp. 2-19.

Laurini R., Servigne S., Favetta F. 2016. An Introduction to Geographic Rule Semantics. In Proceedings of the 22nd International Conference on Distributed Multimedia Systems (DMS 2016), Salerno, Italy, November 25-26, 2016. Published by Knowledge Systems Institute, ISBN: 1-891706-40-3, pp. 9197.

Laurini, R. 2017. Geographic Knowledge Infrastructure: Applications to Territorial Intelligence and Smart Cities. ISTEElsevier. 320 p. April 2017.

Lin H., \& Chen M. 2015. Managing and sharing geographic knowledge in virtual geographic environments (VGEs), Annals of GIS, Volume 21:4, pp. 261-263, DOI: 10.1080/19475683.2015.1099568.

Morgan T. 2008. Business Rules and Information Systems: Aligning IT with Business Goals. Addison-Wesley.

Rogers E. 1995. Diffusion of innovations, Free Press, New York, 4th edition.

Ross R. G. 2011. More on the If-Then Format for Expressing Business Rules: Questions and Answers, Business Rules Journal, Vol. 12, No. 4 (Apr. 2011), URL: http://www.BRCommun 2002ity.com/a2011/b588.html.

Rovira C. 2008. Technology Watch and Competitive intelligence for SEM-SEO. "Hipertext.net", 6, 2008. $<$ http://www.hipertext.net>

Shekhar S., Huang Y. 2001. Discovering spatial co-location patterns: A summary of results. International Symposium on Spatial and Temporal Databases, pp. 236-256. Springer Berlin Heidelberg.

Tobler W. 1970. A Computer Movie Simulating Urban Growth in the Detroit Region". Economic Geography, 46 (2), pp. 234240 . 